SUNY-NTG-94-14

March. 1994

\title{
Where the excess photons and dileptons in SPS nuclear collisions come from?
}

\author{
E. V. Shuryak and L. Xiong \\ Department of Physics \\ State University of New York at Stony Brook \\ Stony Brook, New York 11794
}

\begin{abstract}
Recently the first single photon spectra from CERN energy heavy-ion collisions were reported by WA80, while NA34/3 and NA38 have obtained the spectra for dileptons with the mass up to $4-5 \mathrm{GeV}$. The production rates for photons and dileptons significantly increase when reactions involving the $A_{1}$ meson are included. However, with the conventional expansion scenario, the absolute yields are still significantly smaller than the observed ones. It may indicate that expansion in the "mixed state" takes much more time.
\end{abstract}


Observation of the "penetrating probes", photons and dileptons, was for a long time considered to be the best ways to get information about the early hot stage of nuclear collisions [1], see recent reviews in [2]. Unfortunately, such experiments are difficult, so only recently their first preliminary results were reported.

In this letter we discuss whether one can or cannot explain these observations. The first general question to address is at what stage of the collision the observed photons and dileptons are created. Three qualitatively different stages are generally considered (i) the quark-gluon plasma (QGP) phase; (ii) the mixed stage; and (iii) hadronic stage. Depending on which one is considered, the production rates is then evaluated differently.

At RHIC and LHC energies a production of "hot glue" with $T \sim 2-3 T_{c}$ may provide a window of opportunity to see the QGP signals [3], but at SPS the plasma neither can be significantly hotter than $T_{c}$, nor it may occupy a significant space-time volume: so it will be neglected in this paper.

Speaking about the mixed stage, we use it in a broad sense, as a narrow temperature interval $\Delta T<<T_{c}$ in which the pressure remains nearly constant, but the energy density changes a lot円 from $\epsilon_{\min }$ to $\epsilon_{\max }$, different by about an order of magnitude. With such broad definition, the mixed phase clearly dominates the space-time evolution of the system at CERN SPS energies. Although this fact makes theoretical predictions difficult, a proximity to the phase transition makes the experimental information more challenging and interesting.

Photon production rate in hot hadronic matter was first considered in a pion gas, but then the $\rho$ mesons where shown to be important due to $\pi \rho \rightarrow \pi \gamma$ [7]. Further studies [8] have found that the reaction $\pi \rho \rightarrow A_{1} \rightarrow \pi \gamma$ increase the rate by another factor 3 2. As a result, in the relevant kinematic region the hadronic component of the mixed phase "overshines" the plasma one, even as a rate (per space-time volume).

Let us start with as simple and conventional model of the space-time evolution as possible. We called it the the frozen motion model (FMM), assuming that: (i) rapidity of any matter element is unchanged during the

\footnotetext{
${ }^{1}$ Whether the actual phase transition is first or second order in mathematical sense, or it is just a "rapid crossover", the existence of the mixed phase in this broader sense is supported by all available lattice data on QCD thermodynamics.

${ }^{2}$ Additional small enhancement comes from constructive interference of the $A_{1}$-based and other diagrams [9].
} 
expansion; (ii) there is no transverse expansion; (iii) at the end of the mixed phase, $\epsilon=\epsilon_{\min }$ the pion density corresponds to that of the ideal pion gas; (iv) this moment can be obtained for each rapidity interval from the experimental pion rapidity distribution $\int N_{\pi}(y) / d y$. If so, the longitudinal size of a matter element is $\Delta z=\tau \Delta y$ where $\tau$ is the proper time and $\Delta y$ is its rapidity spread . As a result, the space-time volume spent in the mixed phase by such matter element is

$$
\Delta V_{4}=\Delta y \frac{V_{\pi}^{2}}{2 A_{t}}\left(\frac{d N_{\pi}(y)}{d y}\right)^{2}
$$

where $A_{t}$ is the (projectile) transverse area, and $V_{\pi}$ is the volume per pion at the end of the mixed phase (it depends on the assumed $T_{c}$ and, the upper end of the possible range $T_{c}=180 \mathrm{MeV}$ corresponds to $V_{\pi}=3.7 \mathrm{fm}^{3}$ ). For example, an element in the matter central rapidity $\left(y_{c} \approx 2.6\right.$ for $\mathrm{S}-\mathrm{W}$ collisions) will stay in the mixed phase for the proper time $\tau \approx 10 \mathrm{fm} / \mathrm{c}$, f which is about the same as given by most event generators.

The first statistically significant data on the single photon production were recently reported [5] These data are shown for central events $(7.7 \%$ of the minimum bias cross section) in Fig.1 as a $p_{t}$ spectrum. A striking fact is the "apparent temperature" (a $p_{t}$ slope) is about $3 / 4$ of that for the pions: it fits well the idea that these photons are emitted before the transverse expansion takes place.

Using the rates from [8] and the FMM described above, we get the absolute photon yield at central rapidities. The results, shown in Fig.1., demonstrate that the $T_{c}=180 \mathrm{MeV}$ case fits the slope, but not the magnitude! Which elements of the calculation could then be modified, in order to increase the photon yield?

\footnotetext{
${ }^{3}$ Only at this last point we deviate from the usual scaling (Bjorken) picture, which additionally demands a plateau $d N_{\pi}(y) / d y=$ const. Thus, FMM is its minimal extension to not-so-high SPS energy, at which the end line of the mixed phase (or the freeze-out line) are definitely not hyperbolae in the z-t plane.

${ }^{4}$ One should not confuse this number neither with the freeze-out time (which is about twice larger), nor with the pion emission time as measured by the pion interferometry. The latter depends on the curvature of the decoupling surface, and in general can be different, for example very short, as suggested by available data. [6].

${ }^{5}$ Statistically insignificant excess of photons $(7.5 \pm 11 \%)$ was also reported by CERES
} 
One may question whether at $T \approx T_{c}$ the masses and widths of $\rho, A_{1}$ are still at their vacuum values, or rather, due to chiral symmetry restoration, one may expect $m_{A 1}\left(T \rightarrow T_{c}\right) \rightarrow m_{\rho}\left(T \rightarrow T_{c}\right)$. However, we have found that the photon yield happens to depend weakly on the $A_{1}$ mass: a gain from smaller Boltzmann suppression is compensated by a shift away from the top of the resonance. Similarly, we have found weak sensitivity to the $A_{1}$ width, and conclude that it is impossible to increase the rate by $A_{1}$ modifications alone.

One may now question the conventional FMM picture of space-time evolution. As pointed out long ago [4], the matter in the mixed phase is very soft: its pressure is very low compared to its high energy density. Therefore, one may look at an unconventional scenario for the AGS-SPS energies. Thus we propose a slow expansion model 9 (SEM), in which the observed pion rapidity distribution is formed after the mixed phase is over. If the expansion of the mixed phase is slower than assumed above, more photons can be produced. For example, if a mixed phase expands in $\tau \sim 30-40 \mathrm{fm} / \mathrm{c}$ (instead of 10 $\mathrm{fm} / \mathrm{c}$ in FMM), one can naturally explain the WA80 data. A simple test for this proposal is provided by the following: an excess photons should all be peaked around the central rapidity $y \approx y_{c}$.

Let us now turn to dilepton production. Two muon experiments, NA34 [11] and NA38 [12] have reported the "unaccounted" excess of dileptons in central S-W nuclear collisions, compared to pA and peripheral collisions. The excess is observed for pairs in the invariant mass region $M \sim 1-2 \mathrm{GeV}$, right where the contribution from the mixed phase is expected.

Among the dilepton production mechanisms, the fundamental $\bar{q} q$ annihilation in the QGP phase [1] should be significant for higher masses (and energies). In the hadronic and mixed phase, the $\pi^{+} \pi^{-}$annihilation is the dominant for $M<1 \mathrm{GeV}$, then the $K^{+} K^{-}$annihilation becomes important also. The dilepton production for several processes in hot mesonic gas were calculated in [13], to which other important reactions involving the $A_{1}$ meson were added recently [14]. We have recalculated the rate for the above processes such as $\pi+A_{1} \rightarrow l^{+} l^{-}$with the somewhat different $\pi A_{1} \gamma$ interaction [8] : the results generally agree: the remaining ambiguity is mainly due to

${ }^{6}$ The hydrodynamics of fireball expansion in the corresponding region of initial conditions currently under investigations [10] shows that for some particular initial conditions it is indeed the case. 
uncertain formfactors 7 .

We have calculated the thermal dilepton spectra in the FMM model using $T_{c}=180 \mathrm{MeV}$, see fig.2. The comparisons with the experiments require the acceptance of dileptons in certain rapidity window $[y 1, y 2]$, which is

$$
A(y, M)=\int_{\max \left[0, \frac{\tanh (y-y 1)}{\sqrt{1-4 m_{l}^{2} / M^{2}}}\right]}^{\min \left[1, \frac{\tanh (y 2-y)}{\sqrt{1-m_{2}^{2} / M^{2}}}\right]} f(\cos \theta) d \cos \theta
$$

for the pair with invariant mass $M$ and rapidity $y . \quad f(x)$ is the process dependent angular distribution and is normalized within $x=0,1$. The data reported by NA34 and NA38 lack the absolute normalization, therefore we have used the Drell-Yan process as the benchmark. We demand that our calculated curve 8 goes through the largest mass data points, where DY should definitely dominate. (Our DY evaluation agrees well with the those indicated in the experimental papers.)

The predicted dilepton spectra for three CERN experiments are shown in Fig.2. Like for the photons, a conventional FMM picture does not produce a sufficient yield of dileptons to explain the data. The unconventional SEM can then be the explanation: about the same factor is missing here of.

In order to test it experimentally, one may look at two different things. The first, as for photons, is the unusual rapidity distribution: in SEM one expects larger dilepton excess at central rapidities (NA45) compared to the forward ones (NA34, Na38). The second, specific for dileptons, is a search for the possible "shoulders" in the M-spectra. Of particular interest is the $\pi A_{1}$ threshold: it was recently suggested [16] that if a pion can come from the hypothetical "disoriented chiral condensate", it possesses very small momentum and then the peak develops near the $A_{1}$ mass!

\footnotetext{
${ }^{7}$ The form factors for $\pi \pi, K K$ annihilation are both experimentally determined [15] for $M<2 \mathrm{GeV}$. But it is not known for pi $A_{1}$ annihilation. In particular, its threshold is right below the $\rho^{\prime}(1420)$ resonance, whose contribution to the $\pi A_{1} \gamma$ vertex remains unknown. Following [13, 14] it is assumed to be the same as the pion form factor.

${ }^{8}$ We have scaled the pp collision by $\left(A B^{1 / 3}+A^{1 / 3} B\right) / 2+\left(A^{2 / 3}-B^{2 / 3}\right)^{2} / 4 \ln ((1-$ $\left.\left.(A / B)^{1 / 3}\right) /\left(1+(A / B)^{1 / 3}\right)\right)$ for central $\mathrm{AB}$ collisions. Also we have included the $\mathrm{K}$-factor as 2 .

${ }^{9}$ Let us mention two more puzzles, also coming from recent experiments. Brookhaven experiment E814 has observed a low- $p_{t}$ component in the $K^{+}, K^{-}$spectra, and E802 has found about 5 times more $\Xi$ hyperons than the conventional estimates. The slow expansion scenario at mixed phase may help to explain all of them!
} 
In conclusion, we have evaluated photon and dilepton yields coming from the "mixed phase" created in CERN SPS experiments. We have found, that even taking parameters at their extreme (e.g. take $T_{c}$ at its highest end, etc), one cannot explain the observed photon and dilepton excess in the conventional model which assumes constant velocity of the matter during the expansion. Another proposed scenario, in which the mixed phase fireball expands much slower, during the time of about 30-40 fm/c, can definitely explain them all, and possibly some other puzzles. Simple tests for this exciting scenario are proposed.

\section{Acknowledgements}

We acknowledge interesting discussions of the subject with G.Brown, P.Braun-Munziger and V.Koch. This work is supported in part by the US Department of Energy under Grant No. DE-FG02-88ER40388 and No. DEFG02-93ER40768. 


\section{References}

[1] E.Shuryak Phys.Lett.78B:150,1978, Sov.J.Nucl.Phys.28:408,1978.

[2] P.V.Ruuskanen, Nucl.Phys. A544(1994) 169c. J.Kapusta, Nucl.Phys. A566(1994) 45c.

[3] E. Shuryak, Phys.Rev.Lett. 68 (1992) 3270; E. Shuryak and Li Xiong, Phys.Rev.Lett., 70 (1993) 2241.

[4] E.Shuryak and O.V.Zhirov, Phys.Lett.89B (1979) 253-255; L.van Hove, Z.Phys.C21 (1983) 93.

[5] R.Santo, Nucl.Phys. A566(1994) 61c. Single Photon and Neutral Meson Production from WA80, IKP-MS-93/0701, Munster 1993.

[6] A.Drees, Nucl.Phys. A566(1994) 87c.

[7] J. Kapusta, P. Lichard, and D. Seibert, Phys. Rev., D44 (1991)2774.

[8] Li Xiong, E.Shuryak, G.E.Brown, Phys.Rev.D46 (1992) 3798.

[9] C.Song, Phys.Rev.C, 47 (1993) 2861.

[10] C.M.Hung and E.Shuryak, Hydrodynamics near the QCD phase transition, SUNY-NTG preprint, 1994.

[11] A.Mazzoni,Nucl.Phys. A566(1994) 95c

[12] C.Lourenco,Nucl.Phys. A566(1994) 77c

[13] C. Gale and P. Lichard, Lepton pairs from thermal mesons, McGill/93-8, TPI-MINN-93/19-T.

[14] C.Song, Che Ming Ko and C.Gale, Role of the $a_{1}$ meson in dilepton production from hot hadronic matter. Texas A\&M University preprint.

[15] L. M. Barkov etal, Nucl. Phys. B256 (1985) 365; D. Bisello etal, Phys. Lett. B220 (1989) 321; Ivanov et al. Phys. Lett. B107 (1981) 297.

[16] Z.Huang, M.Suzuki and X.-N.Wang, preprint LBL-35337, 1994. 


\section{Figure Captions}

1. Transverse momentum spectrum of single photons in $200 \mathrm{AGeV} \mathrm{S-Au}$ collisions [5] for central events (7.7\% of the minimum bias cross section). The solid line stands for FMM and $T_{c}=180 \mathrm{MeV}$ (two dotted lines correspond to other choices $T_{c}=140,220 \mathrm{MeV}$ ). The individual contribution from reactions $\pi \pi \rightarrow \rho \gamma$ is shown by short dashed line, and from $\pi \rho \rightarrow A_{1} \rightarrow \pi \gamma$ by the dash-dotted one.

2.Mass spectra of dileptons production evaluated in the FMM model $\left(T_{c}=180 \mathrm{MeV}\right)$ and integrated over the rapidity windows of three CERN experiments are shown by the solid curves. The contributions of $\pi \pi, K^{+} K^{-}, \pi A_{1}$ annihilation are shown by dotted, dash-dotted and short-dash curves, while the Drell-Yan by the long-dash one. The data points are those reported by NA34 and NA38 (We simply took one of the four available sets, 91 SU Psi, others are similar.) 
This figure "fig1-1.png" is available in "png" format from: http://arxiv.org/ps/hep-ph/9404325v1 
This figure "fig1-2.png" is available in "png" format from: http://arxiv.org/ps/hep-ph/9404325v1 